TecnoLógicas

ISSN-p 0123-7799

ISSN-e 2256-5337

Vol. 21, No. 43, pp. 107-125

Sep-dic de 2018

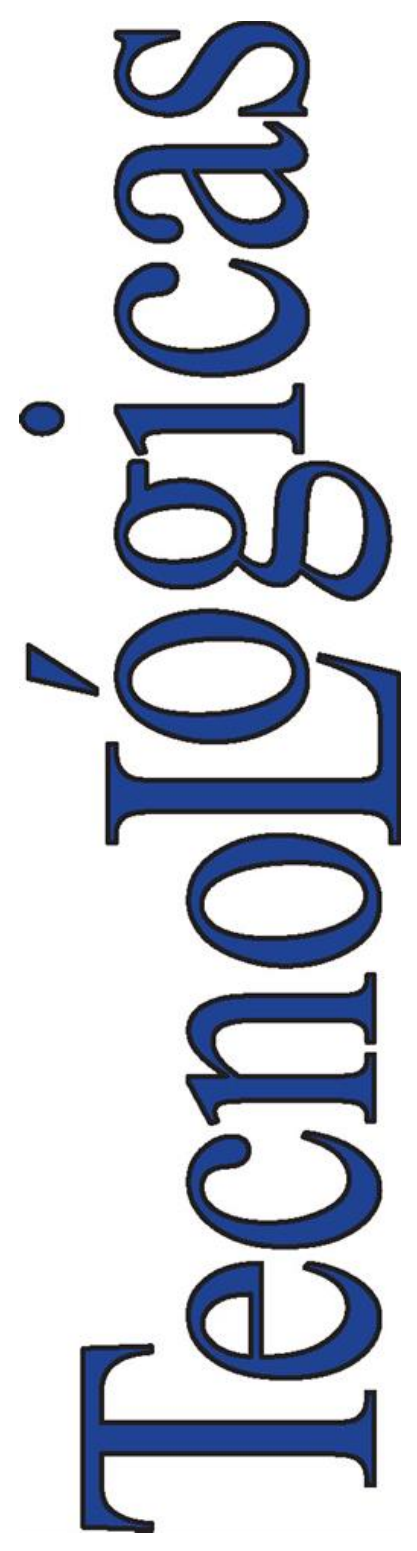

(c) Instituto Tecnológico Metropolitano

Este trabajo está licenciado bajo una Licencia Internacional Creative Commons CC BY-NC-SA

\section{Desarrollo de un prototipo de micro-red residencial a baja escala}

\section{Development of a small-scale residential microgrid prototype}

\author{
Wilmer Guacaneme ${ }^{1}$, Andrés F. Rodríguez ${ }^{2}$, Luis M. \\ Gómez $^{3}$, Francisco Santamaría ${ }^{4}$ y César Trujillo ${ }^{5}$
}

Recibido: 13 de abril de 2018

Aceptado: 03 de septiembre de 2018

Cómo citar / How to cite

W. Guacaneme, A. F. Rodríguez, L. M. Gómez, F. Santamaría, y C. Trujillo, Desarrollo de un prototipo de micro-red residencial a baja escala. TecnoLógicas, vol. 21, no. 43, pp. 107-125, 2018.

1 Ing. Eléctrico, Grupos GCEM y LIFAE, Facultad de Ingeniería, Universidad Distrital Francisco José de Caldas, Bogotá-Colombia, weguacanemem@correo.udistrital.edu.co

2 Ing. Eléctrico, Grupos GCEM y LIFAE, Facultad de Ingeniería, Universidad Distrital Francisco José de Caldas, Bogotá-Colombia, afrodriguezb@correo.udistrital.edu.co

3 Ing. Eléctrico, Grupos GCEM y LIFAE, Facultad de Ingeniería, Universidad Distrital Francisco José de Caldas, Bogotá-Colombia, pgomezlm@correo.udistrital.edu.co

4 PhD. en Ingeniería, M.SC en Ingeniería Eléctrica, Ing. Electricista, Profesor Asociado, Investigador Grupo GCEM, Facultad de Ingeniería, Universidad Distrital Francisco José de Caldas, Bogotá-Colombia, fsantamariap@udistrital.edu.co

5 PhD. en Ingeniería Electrónica, M.SC en Ingeniería Eléctrica, Ing. Electrónico, Profesor Titular, Investigador Grupo LIFAE, Facultad de Ingeniería, Universidad Distrital Francisco José de Caldas, BogotáColombia, cltrujillo@udistrital.edu.co 


\title{
Resumen
}

Este artículo presenta el desarrollo de un emulador de una Micro-Red (MR) eléctrica residencial aislada, que incluye generación distribuida, en este caso fotovoltaica, almacenamiento de energía mediante baterías, y tecnología de vehículos eléctricos que permite suministrar energía a la red eléctrica (V2G). Se realizó un estudio para establecer las principales cargas en una instalación residencial y la prioridad que los usuarios le dan a cada una de ellas. Adicionalmente, se implementó un Sistema de Gestión Energética (SGE), el cual establece los criterios y parámetros de selección para determinar la fuente de energía adecuada en un momento dado y la operación de las cargas dependiendo de los recursos energéticos disponibles. Además, se presenta el diseño y dimensionamiento de los elementos de potencia que permiten suministrar energía a las cargas de la vivienda. Una vez implementado el emulador de micro-red residencial aislada, se realizaron diferentes pruebas, las cuales permiten verificar el correcto desempeño de los elementos del prototipo y del sistema de gestión de energía implementado. Los resultados permiten evidenciar que el emulador desarrollado es una poderosa herramienta académica y de investigación que permite la ejecución de múltiples pruebas y montajes experimentales que facilitan la comprensión y validación de cálculos y aproximaciones teóricas en el campo de micro-redes eléctricas aisladas.

\section{Palabras clave}

Almacenamiento de energía, emulador, Generación Distribuida (GD), Micro-red (MR), Sistema de Gestión de Energía (SGE), Vehículo a la red (V2G).

\begin{abstract}
This paper presents the development of an isolated residential microgrid (MR) emulator that includes distributed photovoltaic generation, energy storage using batteries, and electric vehicle technology supplying energy to the power grid (V2G). A study established the main loads in a residential facility and the priority that users give to each of them. Additionally, an energy management system (EMS) was implemented to define the selection criteria and parameters to determine the appropriate energy source at a given time and the operation of the loads according to the availability of energy. In addition, this article presents the design and sizing of the power elements that allow to supply energy to the residential loads. Once the emulator of the isolated residential microgrid was implemented, several laboratory tests were conducted. They enabled to verify the correct operation of the elements of the prototype and the implemented energy management system. The results show that the emulator developed in this work is a powerful academic and research tool that allows multiple tests and experimental setups that facilitate the understanding and validation of calculations and theoretical approximations in the field of isolated electrical microgrids.
\end{abstract}

\section{Keywords}

Energy storage, Emulator, Distributed Generation (DG), Microgrid (MG), Energy Management System (EMS), Vehicle to Grid (V2G). 


\section{SIGLAS}

A continuación, se presentan las siglas empleadas en este artículo para facilitar su comprensión.

CI: Carga diaria de corriente

CNS: Capacidad Nominal del Sistema

D: $\quad$ Ciclo útil

GD: Generación Distribuida

FV: $\quad$ Sistema Fotovoltaico

MPP: Punto de Máxima Potencia

MR: Micro-red

$\mathrm{P}_{0}$ : $\quad$ Potencia de salida

PIN: Potencia de entrada

Ri: Resistencia aparente del panel FV

$\mathrm{R}_{\mathrm{MPP}}$ : Resistencia en el máximo punto de potencia

SGE: Sistema de Gestión Energética

SOC: Estado de carga de la batería

TO: Tiempo de Operación

VE: Vehículo Eléctrico

V2G: Vehículo eléctrico suministrando energía a la red

$\mathrm{V}_{0}$ : Tensión de salida

$\mathrm{V}_{\text {IN: }}$ Tensión de entrada

VFv: Tensión de la batería del sistema fotovoltaico

VvE: Tensión de la batería del vehículo eléctrico 


\section{INTRODUCCIÓN}

En las últimas décadas se ha comprobado que el uso de combustibles fósiles empleados en la generación de energía eléctrica, produce varios problemas desde el punto de vista social, económico y ambiental [1]. De otro lado, los países con alta dependencia en las fuentes hídricas para la producción de energía, se han visto afectados por fuertes sequías y cambios abruptos en el clima, a causa de fenómenos naturales y el cambio climático, lo cual ha dejado en evidencia la vulnerabilidad de estos países al depender principalmente de una única fuente de energía. Debido a lo anterior, las fuentes de generación con energías renovables han surgido como una alternativa y complemento a las fuentes convencionales de energía.

Las Micro-redes (MR) eléctricas plantean el uso de las energías renovables para suplir la demanda de energía. Estos son sistemas que integran fuentes de energía convencional y no convencional, dispositivos de almacenamiento de energía y cargas capaces de autorregularse, para suplir la demanda en lugares localizados cerca a la generación [2]. Las MR permiten la utilización de fuentes de Generación Distribuida (GD) basadas en Sistemas Fotovoltaicos (FV), eólicos, cogeneración con microturbinas de gas, etc., localizadas cerca a los usuarios finales, incluso se han integrado los Vehículos Eléctricos (VE) como cargas o como fuentes, lo que permite mejorar los indicadores de eficiencia, calidad y confiabilidad [3].

Actualmente se busca el cambio de los medios de transporte convencionales, soportados fundamentalmente en combustibles fósiles, por medios más amigables como el VE. Además, el modo de operación (V2G) permite a los usuarios emplear la energía almacenada en las baterías para cubrir parte de la demanda de energía de un sistema, especialmente la requerida por cargas prioritarias. En esta configuración, el VE conectado a la red puede inyectar a la red la energía almacenada en la batería o realizar la recarga de la misma [4]. No obstante, la inclusión masiva de VE al sistema eléctrico puede provocar un cambio drástico en la curva de demanda de energía eléctrica, lo cual causaría impactos técnicos y económicos sobre la red [5], [6].

Un Sistema de Gestión de Energía (SGE), se basa en el monitoreo de diferentes variables dentro de una red eléctrica, a partir de las cuales emplea algoritmos para la toma de decisiones que aseguren la correcta operación de dicha red. Los SGE son fundamentales en el funcionamiento de las micro-redes, ya que procesan los datos, y realizan el seguimiento y control del centro de despacho de la micro-red [7], [8]. Además, mediante los sistemas de comunicación y medida se recopilan datos de los diferentes dispositivos de la instalación, como lo son la disponibilidad de GD y el consumo de las diferentes cargas, con la cual el SGE puede realizar las acciones requeridas [9].

En resumen, el estudio de micro-redes requiere de la implementación de GD, cargas, sistemas de comunicación, dispositivos de control, elementos de medida y estrategias de gestión, con el fin de caracterizar y ajustar la micro-red a los requerimientos de los usuarios. Asimismo, la inclusión del VE como carga o fuente de energía requiere de una infraestructura adicional y una capacidad de energía mayor por parte del sistema al que se conecta.

En este artículo se presenta el desarrollo e implementación de un emulador de una micro-red residencial operando de manera aislada, el cual interactúa con un sistema de gestión de energía y contempla el uso de tecnología V2G. La ventaja de este emulador es que permite realizar los estudios de las microredes eléctricas con niveles de corriente y potencia menores que en una micro-red real. 
Inicialmente, se presenta la metodología abordada para el desarrollo e implementación del emulador, a partir de las características eléctricas de la microred, describiendo el diseño de los elementos que la componen, además se presenta el sistema de gestión implementado y las pruebas realizadas al emulador. La sección 3 aborda los principales resultados del trabajo, a partir de los cuales se discuten los principales aportes del artículo. Finalmente, se presentan las conclusiones del trabajo.

\section{METODOLOGÍA}

En esta sección se presentan las principales características de la micro-red residencial diseñada, a partir de la cual se implementó el prototipo de emulador de micro-red. Posteriormente, se describen los principales elementos del sistema de gestión propuesto y finalmente se describen las pruebas realizadas para verificar el correcto funcionamiento del emulador y del sistema de gestión.

\subsection{Características del emulador de micro- red}

Dentro de las micro-redes aisladas es posible identificar diferentes topologías de diseño y construcción, en este trabajo se estableció que la topología seleccionada debe cumplir con las siguientes características: 1) La fuente principal de alimentación de la micro-red debe ser una fuente de generación alternativa. 2) Integrar un sistema de acumulación de energía que asegure la autonomía de la micro-red. 3) Incluir una batería que represente un VE como carga o fuente de energía de respaldo de la micro-red. 4) Suministrar energía a las cargas en corriente alterna. Además, se deben acondicionar los diferentes niveles de tensión DC para el correcto funcionamiento de los elementos de la micro-red.

La topología que cumple con las condiciones descritas se presenta en la Fig. 1. Dentro de sus características principales se encuentran el uso de un panel fotovoltaico como fuente principal de generación; dos niveles de tensión DC impuestos por el conjunto regulador batería FV, VE, y tensión de operación del inversor; un nivel de tensión AC donde se conectan todas las cargas. Además, permite el uso del VE como fuente de respaldo al suministrar la energía de la batería del VE al barraje.

\subsection{Potencia demandada por la micro-red}

En el diseño de sistemas soportados por energía solar, es necesario conocer la demanda de energía a la cual se va a someter la instalación, así como los hábitos de consumo para determinar la naturaleza de las cargas, su nivel de tensión, número de artefactos y energía consumida por cada uno de ellos.

Existen diferentes trabajos de índole académico [10], [11], [12], corporativo [13] y gubernamental [14], [15], [16], que han planteado estrategias para determinar el comportamiento de la demanda de energía eléctrica a nivel residencial e incluso han evaluado la influencia de los diferentes electrodomésticos en el consumo específico de los usuarios. Los resultados de estos trabajos permitieron identificar algunos de los dispositivos eléctricos de mayor relevancia en el hogar y la importancia que les dan los usuarios a dichos dispositivos. Sin embargo, con el fin de ampliar esta información los autores realizaron una encuesta en la cual se indagó qué artefactos o electrodomésticos deberían incluirse dentro de una vivienda de nivel socioeconómico medio - alto (estrato 4 en Colombia), y cuál es su tiempo de uso, lo cual permitió determinar las cargas conectadas a la micro-red. 


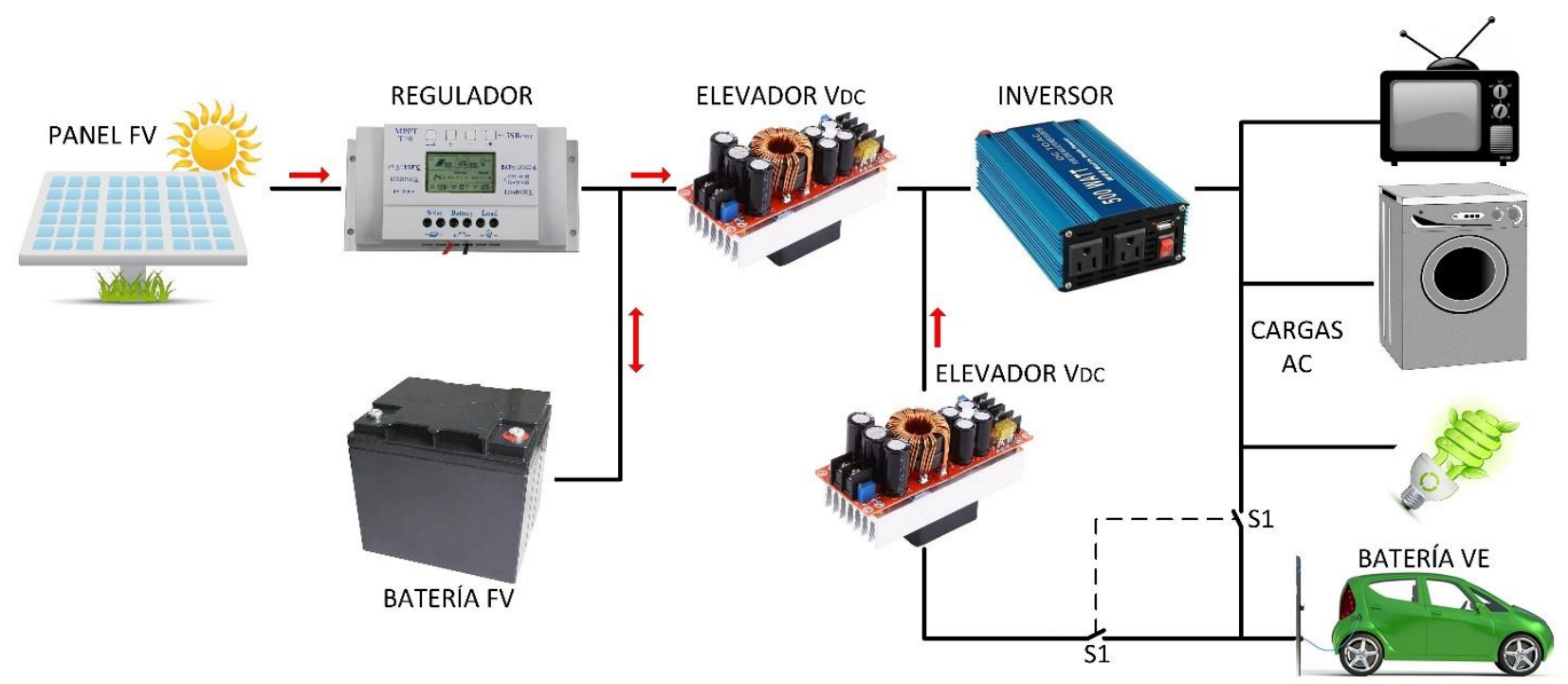

Fig. 1. Topología de la Micro-red. Fuente: autores.

Esta encuesta fue realizada siguiendo el procedimiento propuesto en [17] a una muestra de 85 usuarios, con un intervalo de confianza del $90 \%$ y un error del $5 \%$. Como resultado del proceso, se identificaron los principales electrodomésticos y las diferentes horas del día en que son usados. Posteriormente, se seleccionaron los 9 artefactos de mayor relevancia y se adicionó un circuito para alimentar el vehículo eléctrico, para conformar 20 circuitos en la vivienda con una potencia total de $6039 \mathrm{~W}$ (Tabla 1).

Para poder representar la potencia demandada de la micro-red en el emulador, es necesario realizar un escalamiento de la misma. El factor de escala se determina a partir de la potencia máxima del inversor, $(150 \mathrm{~W})$. Por lo tanto, $150 \mathrm{~W}$ en el emulador equivalen a $6039 \mathrm{~W}$ reales o lo que es igual, $1 \mathrm{~W}$ real corresponde a 24,83 $\mathrm{mW}$ en el emulador de la micro-red.

Para establecer el valor de potencia escalada y el correspondiente factor de escala, no se tuvo en cuenta el factor de demanda empleado en instalaciones eléctricas de acuerdo con lo establecido en la norma NTC 2050 [18] y que se define como la relación entre la máxima demanda y la carga total instalada, es decir, es un indicador del grado de simultaneidad de la carga conectada. Este factor no se tuvo en consideración dentro de los valores de la Tabla 1, ya que el emulador a baja escala desarrollado tiene fines académicos, por lo que dentro de las pruebas que se pueden llevar a cabo es posible que se conecten y alimenten de manera simultánea todas las cargas, lo que pondría en riesgo la instalación o llevaría a que actuaran las protecciones, por lo que el diseño se llevó a cabo previendo la condición crítica.

\subsection{Fuente alternativa de generación}

Para obtener un modelo de micro-red basado en características de operación de equipos reales, el dimensionamiento del emulador se llevó a cabo con dispositivos comúnmente encontrados en el mercado, además en esta investigación se contaba con una fuente con capacidad nominal de $760 \mathrm{~W}$, que permite realizar barridos de tensión entre 0 y $80 \mathrm{~V}$, y adicionalmente permite controlar la corriente en un rango entre 0 y $9,5 \mathrm{~A}$.

Con estos datos se buscó un panel fotovoltaico con corriente de corto circuito cercana a los 9,5 A y que además, bajo condiciones nominales de operación, sea capaz de suministrar la potencia máxima escalada demandada por un usuario, así 
como las posibles pérdidas que se presentan durante la transformación de energía. Por lo tanto, se seleccionó un panel con las características que se presentan en la Tabla 2.

\subsection{Regulador de carga}

Este dispositivo permite realizar la conexión entre el grupo generador (panel batería FV) y el resto del sistema. Además de tener en cuenta las condiciones climáticas, para extraer la máxima potencia de un panel FV, el sistema de conversión de energía se debe llevar al Máximo Punto de Potencia (MPP). Para el caso del convertidor $\mathrm{DC} / \mathrm{DC}$, el regulador ajusta la Ri del panel FV para que sea igual a la $\mathrm{R}_{\text {MPP }}$ [19]. La Tabla 3 presenta una comparación entre algunos convertidores que podrían realizar la función de regulador de carga.

Tabla 1. Potencia real y escalada de los electrodomésticos seleccionados. Fuente: autores.

\begin{tabular}{|c|c|c|c|c|}
\hline Dispositivo & Potencia unitaria (W) & Cant. & Potencia total (W) & Potencia escalada (W) \\
\hline Luminaria comedor & 40 & 2 & 80 & 1,98 \\
\hline Luminaria sala & 40 & 2 & 80 & 1,98 \\
\hline Luminaria pasillo & 35 & 1 & 35 & 0,86 \\
\hline Luminaria cocina & 35 & 1 & 35 & 0,86 \\
\hline Luminaria dormitorio $\mathrm{P}$ & 35 & 2 & 70 & 1,73 \\
\hline Luminaria dormitorio A & 35 & 1 & 35 & 0,86 \\
\hline Luminaria estudio & 40 & 2 & 80 & 1,98 \\
\hline Luminaria baño & 40 & 1 & 40 & 0,99 \\
\hline Televisor sala & 62 & 1 & 62 & 1,53 \\
\hline Televisor principal & 62 & 1 & 62 & 1,53 \\
\hline Equipo de sonido & 120 & 1 & 120 & 2,97 \\
\hline Consola videojuegos & 60 & 1 & 60 & 1,48 \\
\hline Nevera & 300 & 1 & 300 & 7,49 \\
\hline Lavadora & 800 & 1 & 800 & 19,86 \\
\hline Computador 1 & 90 & 1 & 90 & 2,23 \\
\hline Computador 2 & 90 & 1 & 90 & 2,23 \\
\hline Microondas & 700 & 1 & 700 & 17,38 \\
\hline Secador de pelo & 800 & 1 & 800 & 19,84 \\
\hline Circuito especial 1 & 600 & 1 & 600 & 14,89 \\
\hline \multirow[t]{2}{*}{ Vehículo eléctrico } & 1900 & 1 & 1900 & 47,17 \\
\hline & & Total & 6039 & 150 \\
\hline
\end{tabular}

Tabla 2. Características del panel seleccionado. Fuente: autores.

\begin{tabular}{lc}
\hline Característica & Valor \\
\hline Máxima potencia & $150 \mathrm{Wp}$ \\
Tensión en circuito abierto & $22,8 \mathrm{~V}$ \\
Tensión MPP & $18,4 \mathrm{~V}$ \\
Corriente en corto circuito & $8,85 \mathrm{~A}$ \\
Corriente MPP & $8,37 \mathrm{~A}$ \\
\hline
\end{tabular}


Desarrollo de un prototipo de micro-red residencial a baja escala

Tabla 3. Comparación de convertidores. Fuente: autores.

\begin{tabular}{|c|c|c|c|c|}
\hline CONVERTIDOR & Buck-Boost & Cuk & Positive Buck-Boost & SEPIC \\
\hline Polaridad tensión de salida & Invertida & Invertida & Sin invertir & Sin invertir \\
\hline Corriente de entrada & Pulsante & No pulsante & $\begin{array}{l}\text { Dependiente del modo } \\
\text { de operación }\end{array}$ & Pulsante \\
\hline Switch Drive & Flotante & Flotante & Flotante y aterrizado & Aterrizado \\
\hline Eficiencia & Baja & Media & $\begin{array}{l}\text { Alta en el estado } \\
\text { activo }\end{array}$ & Media \\
\hline Costo & $\begin{array}{l}\text { Medio por el } \\
\text { controlador } \\
\text { flotante }\end{array}$ & $\begin{array}{l}\text { Medio por } \\
\text { el capacitor } \\
\text { adicional }\end{array}$ & $\begin{array}{l}\text { Alto por el interruptor } \\
\text { adicional y el } \\
\text { controlador de } \\
\text { interruptor }\end{array}$ & $\begin{array}{l}\text { Medio por } \\
\text { el capacitor } \\
\text { adicional }\end{array}$ \\
\hline
\end{tabular}

Se seleccionó el convertidor SEPIC como regulador de carga, ya que permite conectar el dispositivo de conmutación sólidamente a tierra, no invierte la tensión de salida, es posible aprovechar al máximo la energía disponible y salir del punto de máxima potencia. En la Fig. 2 se muestra la topología del regulador de carga.

Donde la Potencia máxima de salida (P_MAX) y la potencia nominal de entrada (PIN) son $150 \mathrm{~W}$, la tensión de salida $\left(\mathrm{V}_{0}\right)$ es de $12 \mathrm{~V}$. La tensión de entrada $\left(\mathrm{V}_{\text {IN }}=18,4\right.$ V) depende de la tensión en máxima potencia del módulo solar ( $\mathrm{VMP})$. La tensión de entrada máxima (Voc del módulo solar) es $22,8 \mathrm{~V}$ y la tensión de entrada mínima es $10 \mathrm{~V}$. A partir de estos parámetros se tiene un ciclo útil (D) de 0,56, corriente de salida $\mathrm{I}_{0}=12,5 \mathrm{~A}$, la corriente de entrada se definió de acuerdo con la máxima corriente de salida de la fuente Agilent IN $=9,5 \mathrm{~A}$, resistencia de carga mínima $\mathrm{R}_{\text {LOAD_MIN }}=0,96 \Omega, \mathrm{L}_{1}=\mathrm{L}_{2}=98,28 \mu \mathrm{H}, \mathrm{C}_{1}=$ $\mathrm{C}_{2}=466,66 \mu \mathrm{F}$.

\subsection{Sistema de almacenamiento de energía}

Para poder proporcionar autonomía a la micro-red aislada, en caso de presentarse baja irradiancia, se incluyó un banco de baterías capaz de suplir la demanda energética del usuario. Inicialmente, se debe definir el tiempo en el que el prototipo emulará un día de operación, el cual en este caso se estableció en 1,6 horas, es decir, que 4 minutos del emulador representan una hora de consumo $(\mathrm{TO}=$
1,6 h). El criterio para la selección de este tiempo fue la facilidad de realizar pruebas equivalentes a un día en menos de 2 horas, esto con el fin de que el prototipo desarrollado, además de emplearse en actividades de investigación, también se pueda emplear en algunas prácticas en espacios académicos de maestría, por lo que tener tiempos de operación más prolongados limitaría el número de prácticas que se pueden realizar; además, un mayor tiempo de operación hace que se requieran sistemas de almacenamiento de mayor capacidad, lo cual va en contra del objetivo de que el emulador sea portátil y modular.

La capacidad nominal del sistema (CNS) se calcula de la siguiente manera (1) y (2):

$C I=\frac{\text { Energia a suministrar }}{\text { Tensión nominal del sistema }}$
$C I=\frac{60,63 \mathrm{Wh}-\mathrm{TO}}{12 \mathrm{~V}}=5,052 \mathrm{Ah}-\mathrm{TO}$

$C N S=C I \times \#$ dias autonomia

$C N S=5,052 \times 1=5,0527 \mathrm{Ah}-\mathrm{TO}$

Teniendo en cuenta la profundidad de la descarga de cada una de las baterías es necesario aplicar un factor de corrección, el cual está determinado por (3):

$$
\begin{aligned}
& \text { CNS_corr }=\frac{C N S}{\text { Profundidad Descarga }} \\
& \text { CNS_corr }=\frac{5,0527}{0,8}=6,3158 \mathrm{Ah}-\mathrm{TO}
\end{aligned}
$$




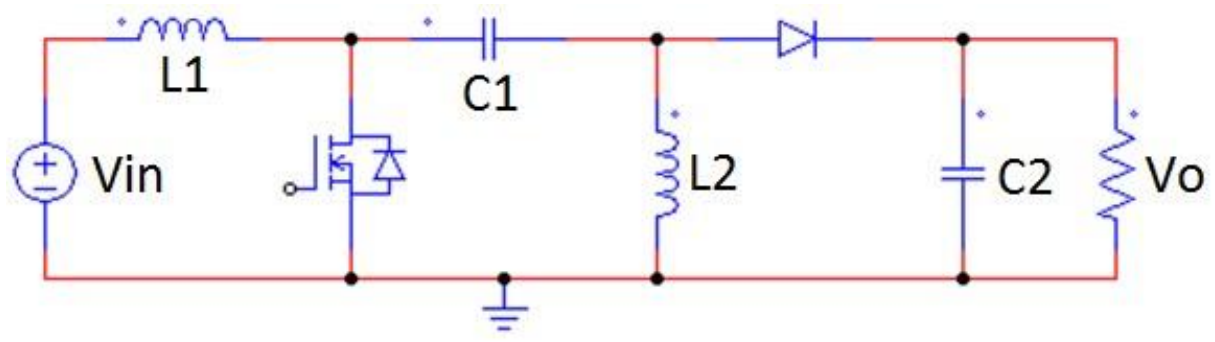

Fig. 2. Topología del regulador de carga. Fuente: autores.

En este caso, se seleccionó una batería de Plomo-Ácido de $12 \mathrm{~V}$ y una capacidad de $7 \mathrm{Ah}$, la cual cumple con las características de diseño previamente señaladas. Para adecuar el nivel de tensión de las baterías del sistema FV al nivel de operación del inversor se utiliza un convertidor Boost. En la Fig. 3 se observa la topología.

En donde $\mathrm{V}_{\text {IN }}=12 \mathrm{~V}$ es la tensión de entrada, $\mathrm{V}_{\mathrm{O}}=24 \mathrm{~V}$ es la tensión de salida, $\mathrm{P}_{\mathrm{O}}=150 \mathrm{~W}$ es la potencia de salida y $\mathrm{F}_{\mathrm{C}}=$ $20 \mathrm{kHz}$ corresponde a la frecuencia de conmutación del convertidor. A partir de estos parámetros se tiene un ciclo útil (D) de 0,5 , resistencia de carga $R=3,84 \Omega$, $\mathrm{L}_{\text {MIN }}=15 \mu \mathrm{H}, \mathrm{C}=651 \mu \mathrm{F}$, porcentaje de rizado $=1 \%, \mathrm{I}_{\mathrm{L}}=8,68 \mathrm{~A}, \mathrm{I}_{\mathrm{L} \_\mathrm{MAX}}=16,6 \mathrm{~A}$, $\mathrm{I}_{\mathrm{L} \_\mathrm{MIN}}=0,6 \mathrm{~A}$.

\subsection{Módulo de carga}

Emula el comportamiento de la demanda eléctrica de la vivienda a partir del encendido y apagado de las 20 cargas resistivas que representan los 20 circuitos presentados en la Tabla 1. Este módulo permite realizar el cierre y la apertura de circuitos a partir de las señales emitidas por un microcontrolador, por tanto, puede emular una curva de demanda a partir de parámetros programados o manuales. Con el fin de emular el comportamiento de un sistema de generación y consumo aislado a partir de fuentes no convencionales de energía, en este artículo se empleó el módulo de carga programando un perfil de demanda preestablecido, como se observa en la sección 3.3.

Las cargas del emulador reciben la orden de conexión o desconexión a partir del sistema de gestión de energía, que habilita o deshabilita el uso de dichas cargas de acuerdo con los criterios descritos en la sección 3.2. (etapa azul de la Fig. 4).

La etapa naranja de la Fig. 4 corresponde al circuito de potencia de la micro-red. Esta etapa cuenta con un interruptor que permite encender cargas de manera manual y un relé que, a partir de la señal del microcontrolador, permite la circulación de corriente hacia el resistor.

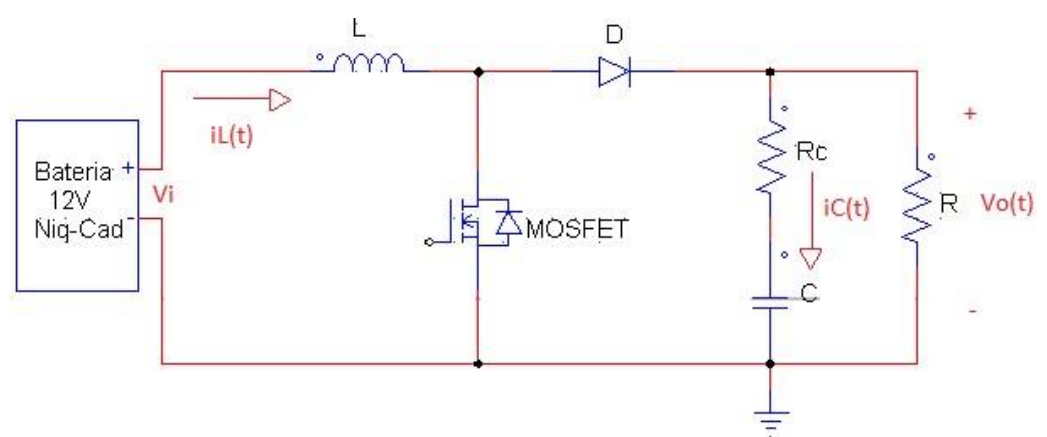

Fig. 3. Topología del Convertidor Boost del sistema FV. Fuente: autores. 


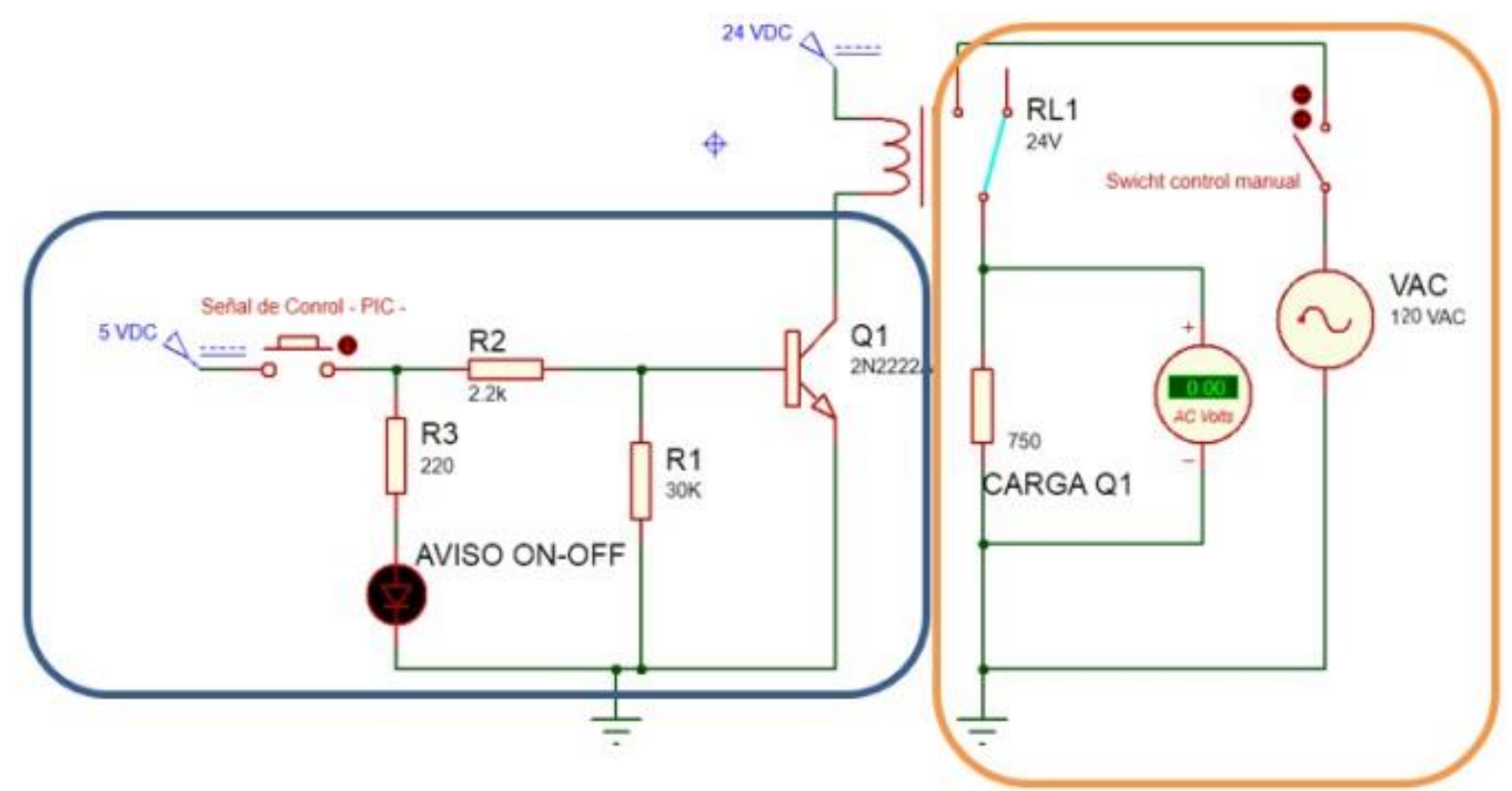

Fig. 4. Circuito de control de cargas. Etapa azul: control y disparo. Etapa naranja: potencia. Fuente: autores.

\subsection{Vehículo eléctrico}

La micro-red propuesta facilita la integración de la tecnología V2G, mediante la cual se puede recargar la batería del VE y además contar con ella como fuente de suministro de energía para el sistema [20], [21]. La Fig. 5 muestra las curvas características de tensión y corriente correspondiente a la recarga de las baterías de algunos de los VE más representativos en el mercado [22]. El objetivo de la batería que se emplee en el emulador, es reproducir el comportamiento de las baterías reales usadas en VE, pero manteniendo los niveles de tensión y corriente dentro de los rangos establecidos para el emulador. En este sentido, empleando el software PSIM se simula el modelo de batería disponible en el paquete Matlab - Simulink, para una batería de 12 V y 7 Ah (Fig. 6).

Comparando las características de la recarga de las baterías de los VE de la Fig. 5 y la batería a emular (Fig. 6), se observa que la batería de menor capacidad y menor tensión seleccionada reproduce adecuadamente el comportamiento de las baterías de los $\mathrm{VE}$, con una tensión casi constante y la caída exponencial de la corriente durante la recarga, por supuesto en un tiempo mucho menor, lo cual es uno de los principios de funcionamiento del emulador.

Cuando el vehículo está en el modo en el cual suministra energía a la micro-red, se emplea un convertidor Boost (Fig. 3) para entregar la energía al barraje DC correspondiente, en donde $\mathrm{V}_{\mathrm{IN}}=13,5-11$ $\mathrm{V}$ es la tensión de entrada, $\mathrm{V}_{\mathrm{O}}=25 \mathrm{~V}$ es la tensión de salida, $\mathrm{P}_{0}=23-15 \mathrm{~W}$ es la potencia de salida y $\mathrm{F}_{\mathrm{C}}=20 \mathrm{kHz}$ corresponde a la frecuencia de conmutación del convertidor. A partir de estos parámetros se tiene un ciclo útil (D) de 0,52 , resistencia de carga $R=41,66 \Omega$, LMIN $=150 \mu \mathrm{H}, \mathrm{C}=220 \mu \mathrm{F}$, porcentaje de rizado $=1 \%, \mathrm{I}_{\mathrm{L} \_\mathrm{MAX}}=16,6 \mathrm{~A}, \mathrm{I}_{\mathrm{L} \_\mathrm{MIN}}=0,6 \mathrm{~A}$.

En el modo carga, y con el fin de representar la demanda de la recarga de la batería, se desarrolló un cargador cuyas características son: potencia máxima: 44,5 W, tensión: $13,5 \mathrm{~V}$, corriente máxima: 3,3 A. 

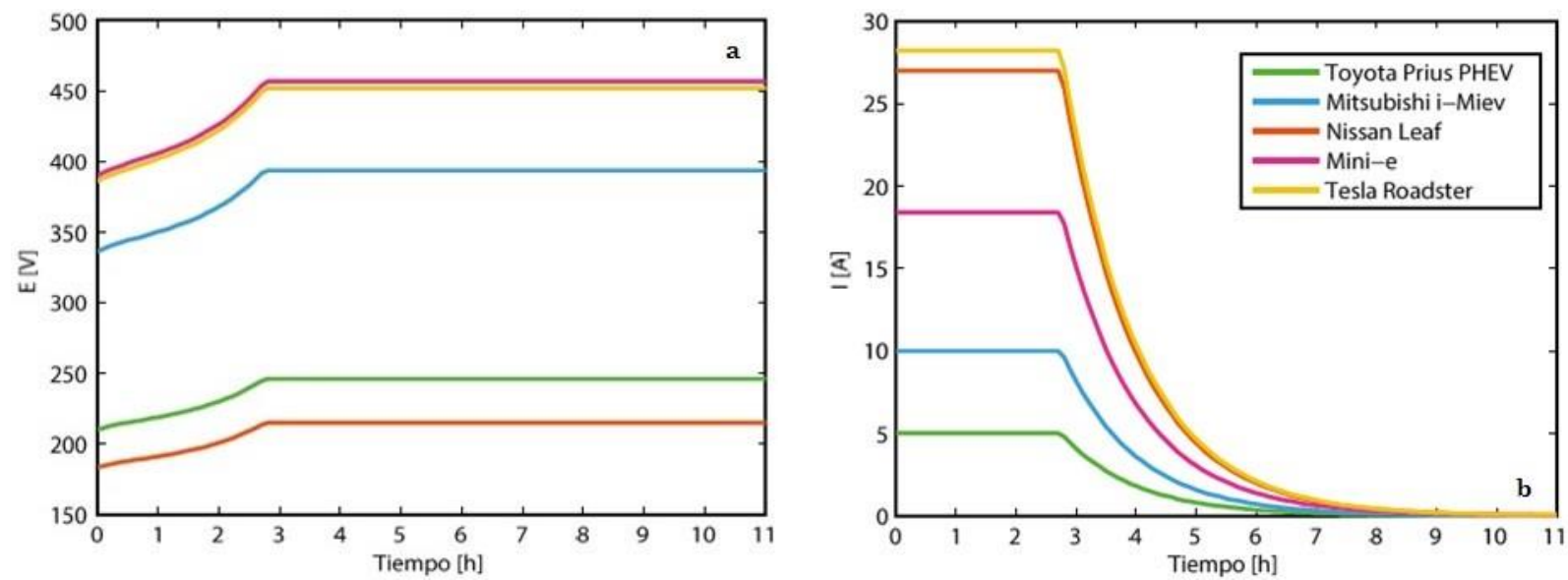

Fig. 5. Característica de recarga de algunos VE Comerciales, a. Tensión, b. Corriente. Fuente: adaptada de [22].
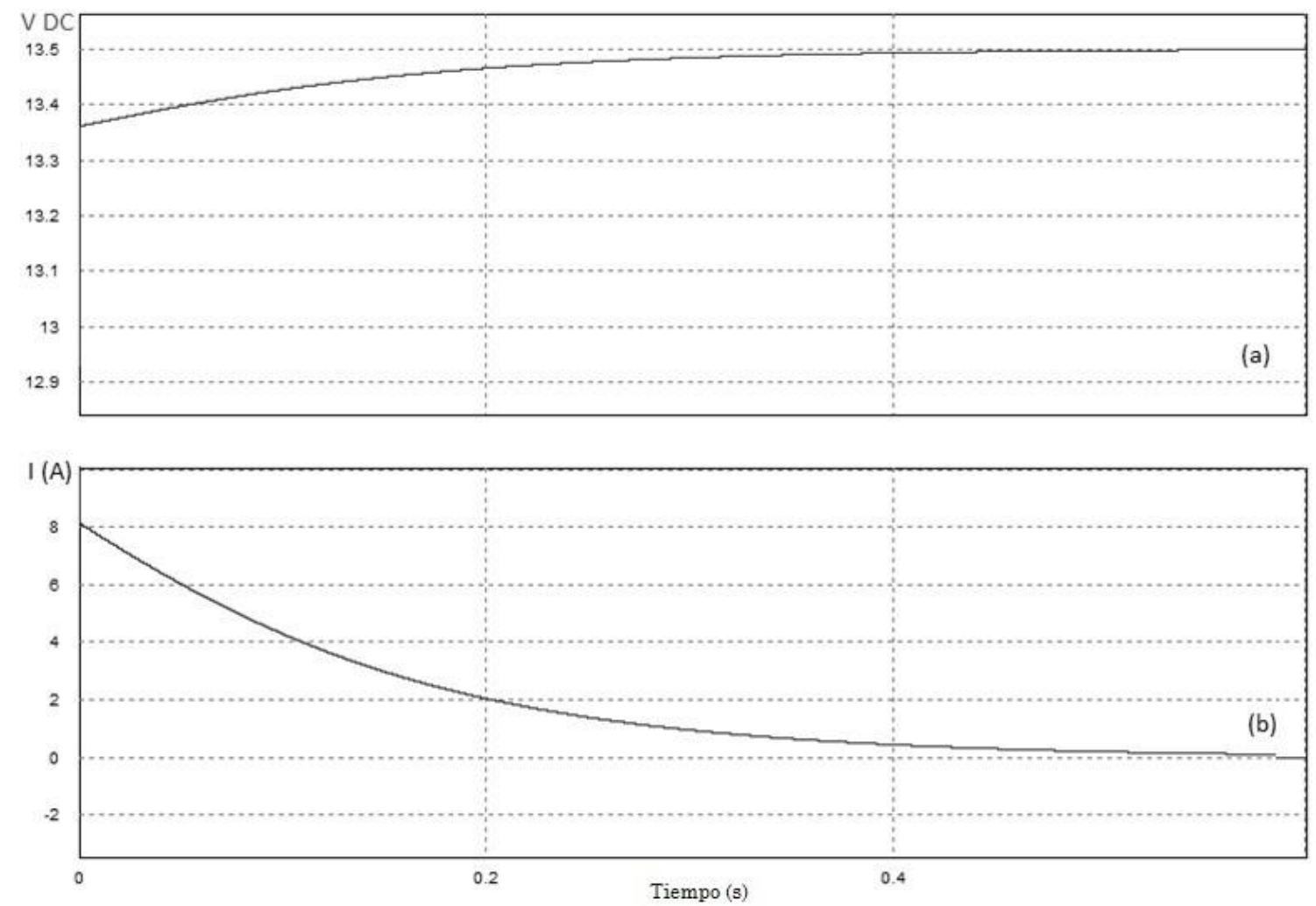

Fig. 6. Características de recarga de la batería para emular el VE. (a) Tensión, (b) Corriente. Fuente: autores.

\section{RESULTADOS Y DISCUSIÓN}

Una vez determinados los elementos que componen el emulador de la micro-red y realizado el diseño correspondiente, en esta sección se presenta la descripción del emulador completo, el sistema de gestión energética propuesto $\mathrm{y}$ las pruebas realizadas.

\subsection{Emulador de micro-red eléctrica}

Con el fin de facilitar la construcción del emulador y brindarle mayor flexibilidad, es posible agrupar los elementos que lo conforman en tres secciones (Fig. 7). El módulo principal corresponde a la MR. Este es el núcleo del sistema, allí se recibe energía de las fuentes y se transfiere a las cargas. 


\subsection{Sistema de gestión energética}

El emulador desarrollado opera de manera aislada a la red de distribución (Fig. 7), por lo que se requiere de un sistema que gestione la energía dentro de la micro-red. Para poder lograr esto, inicialmente se identificaron las principales cargas de la vivienda y se clasificaron de acuerdo con la importancia que le dan los usuarios dentro del hogar (Tabla 4).

Esta información fue recopilada a partir de la encuesta realizada por los autores, en donde se le suministró al encuestado un listado de los dispositivos que se encuentran usualmente en una vivienda y se les preguntó cuál o cuáles priorizarían para el suministro de energía en caso de presentarse racionamiento de la misma. Como resultado se obtuvo una clasificación de 11 cargas de prioridad baja $\left(\mathrm{C}_{\mathrm{B}}\right), 7 \mathrm{de}$ prioridad media $\left(\mathrm{C}_{\mathrm{M}}\right)$ y 2 de prioridad alta $\left(\mathrm{C}_{\mathrm{A}}\right)$, como lo muestra la Tabla 4.

En la Fig. 8 se muestra el diagrama de flujo del sistema de gestión energética de la micro-red, el cual fue implementado en un microcontrolador PIC 18F2550.

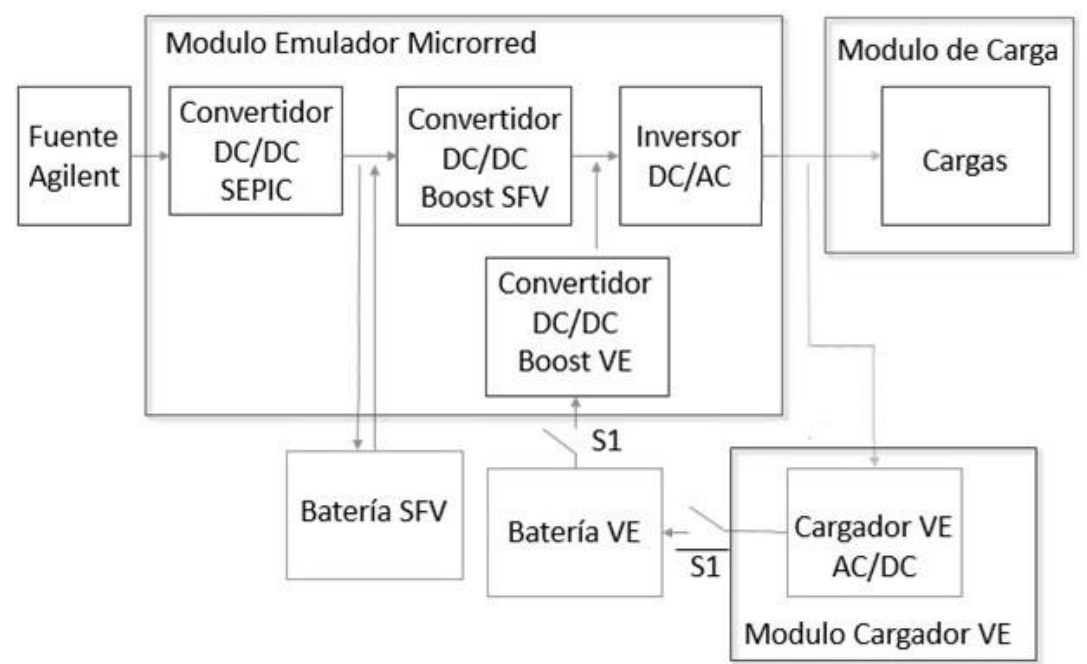

Fig. 7. Topología completa del emulador de la micro-red. Fuente: autores.

Tabla 4. Distribución de Cargas Según Prioridad. Fuente: autores.

\begin{tabular}{ll}
\hline \multicolumn{1}{c}{ Artefacto } & Prioridad \\
\hline Circuito especial 1 & Alta \\
Nevera & Alta \\
Computador 1 & Media \\
Luminaria cocina & Media \\
Luminaria alcoba principal & Media \\
Luminaria baño & Media \\
Luminaria comedor & Media \\
Vehículo eléctrico & Media \\
Televisor sala & Media \\
Luminaria sala & Baja \\
Luminaria pasillo & Baja \\
Luminaria estudio & Baja \\
Computador 2 & Baja \\
Microondas & Baja \\
Lavadora & Baja \\
Televisor alcoba & Baja \\
Equipo de sonido & Baja \\
Video juegos & Baja \\
Luminaria alcoba auxiliar & Baja \\
Secador de pelo & Baja \\
\hline
\end{tabular}




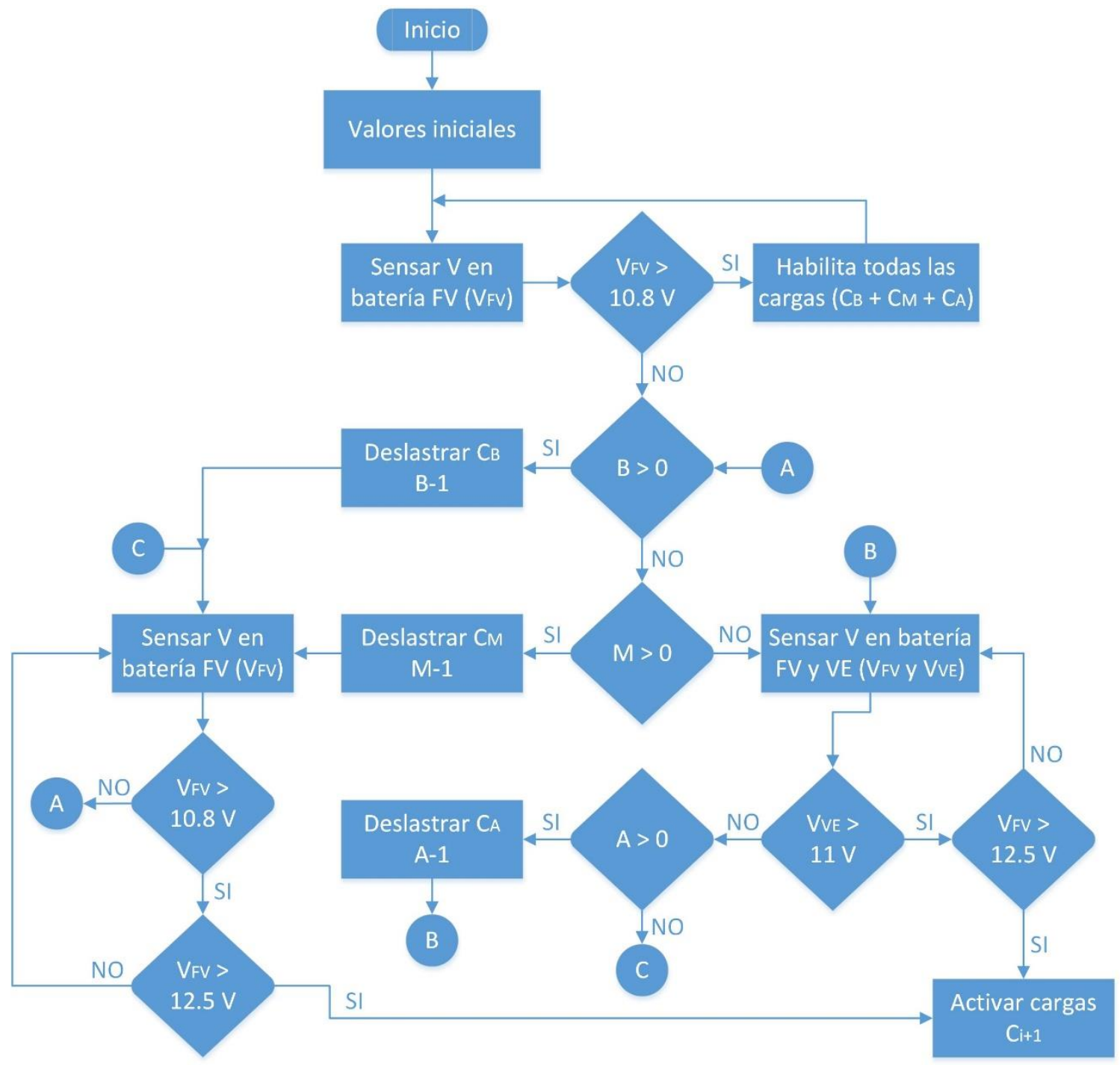

Fig. 8. Diagrama de flujo del sistema de gestión de la micro-red. Fuente: autores.

El primer paso (valores iniciales) es establecer los niveles de tensión de las baterías del sistema FV (VFV) y del VE $\left(\mathrm{V}_{\mathrm{VE}}\right)$, a partir de los cuales el sistema debe suministrar energía de manera continua a las cargas, sin que se produzcan descargas profundas y sin que se comprometa la función principal del VE, que es la movilidad. Los valores seleccionados son 10,8 V para la batería del sistema FV y 11 $\mathrm{V}$ para el VE. Para determinar estos valores, se partió del supuesto de que el estado de carga de la batería (SOC) y la tensión en bornes de la batería son directamente proporcionales, por lo cual se realizó una caracterización de las baterías del emulador, y a partir del modelo Thevenin de la batería se determinaron los valores de tensión para los cuales se tienen niveles de carga por encima del $30 \%-40$ $\%$. Si bien, varios autores han establecido que el comportamiento de la batería es dinámico y la relación entre SOC y tensión no es lineal, también se ha identificado que para valores del SOC por encima del establecido la aproximación lineal arroja resultados aceptables para el análisis que se desea abordar en este artículo [23].

Inicialmente, el sistema de gestión evalúa $\mathrm{VFV}_{\mathrm{FV}}$, y si es mayor a 10,8 V habilita todas las cargas y desactiva el VE como fuente de respaldo. Cuando el nivel de 
tensión cae por debajo de $10,8 \mathrm{~V}$ se inicia un deslastre progresivo de las cargas de baja $\left(\mathrm{C}_{\mathrm{B}}\right)$ y media $\left(\mathrm{C}_{\mathrm{M}}\right)$ prioridad. Esta lógica se repite hasta que se cumpla con la condición establecida para $\mathrm{V}_{\mathrm{FV}}$. Cuando las condiciones de la batería FV determinan que es necesario el deslastre de todas las cargas de baja y media prioridad (los contadores $\mathrm{B}$ y $\mathrm{M}=0$ ), el sistema de gestión puede habilitar el VE como fuente de generación, para esto el sistema sensa $\mathrm{V}_{\mathrm{VE}}$, si es mayor a $11 \mathrm{~V}$ la habilita como fuente de respaldo para alimentar las cargas de alta prioridad $\left(\mathrm{C}_{\mathrm{A}}\right)$. Cuando la tensión cae por debajo de $11 \mathrm{~V}$ el sistema deslastra las cargas de alta prioridad.

Finalmente, cuando las condiciones de irradiancia mejoran y la batería del sistema FV alcanza una tensión de $12,5 \mathrm{~V}$, el sistema de gestión vuelve a activar las cargas deslastradas, deshabilitando el VE como fuente de respaldo.

\subsection{Validación experimental}

Para verificar el funcionamiento del emulador de la micro-red y del sistema de gestión, se realizaron tres pruebas bajo diferentes condiciones de generación, y con la curva de demanda de un día, la cual se determinó a partir de la encuesta realizada por los autores y se programó en el módulo de carga del emulador (Fig. 9).

A continuación, se presentan los resultados medidos en el emulador: potencia generada y potencia demandada medida y se comparan con la potencia demandada estimada de la Fig. 9. Además, se presenta la potencia desarrollada por cada dispositivo del emulador.

Prueba 1. En esta prueba se representó el comportamiento del sistema entre las 18:00 h y las 17:59 h del día siguiente, es decir, que al inicio las condiciones de irradiancia eran nulas (Fig. 10).

La línea punteada corresponde a la demanda estimada, mientras que la línea continua corresponde a la demanda medida. La línea discontinua es la tensión en el barraje de $12 \mathrm{~V}$. La variación observada a las 18:00 $\mathrm{h}$ se debe a un deslastre de cargas $\mathrm{C}_{B}$ para estabilizar el nivel de tensión $\mathrm{V}_{\mathrm{FV}}$.

Entre las 06:00 h y las 17:00 h hay una elevación en la tensión en el barraje de 12 $\mathrm{V}$, a causa de la energía disponible en el sistema FV. En este caso se evidenció el deslastre de carga a partir de la priorización de los circuitos de la vivienda; además, el sistema de gestión reconecta las cargas deslastradas cuando $\mathrm{V}_{\mathrm{FV}}=12,5 \mathrm{~V}$, lo cual ocurrió después de las 07:00 h, como se observa en la Fig. 11.

En donde la línea con marcadores es la potencia de salida del convertidor SEPIC, la línea punteada es la potencia en la batería FV y la línea continua es la potencia demandada por la micro-red.

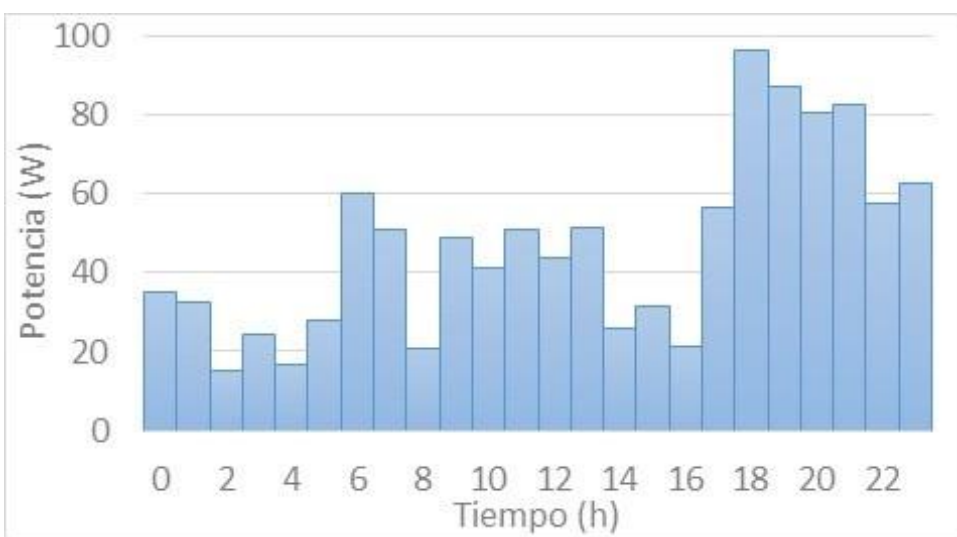

Fig. 9. Potencia demandada estimada construida a partir de la encuesta. Fuente: autores. 
Desarrollo de un prototipo de micro-red residencial a baja escala

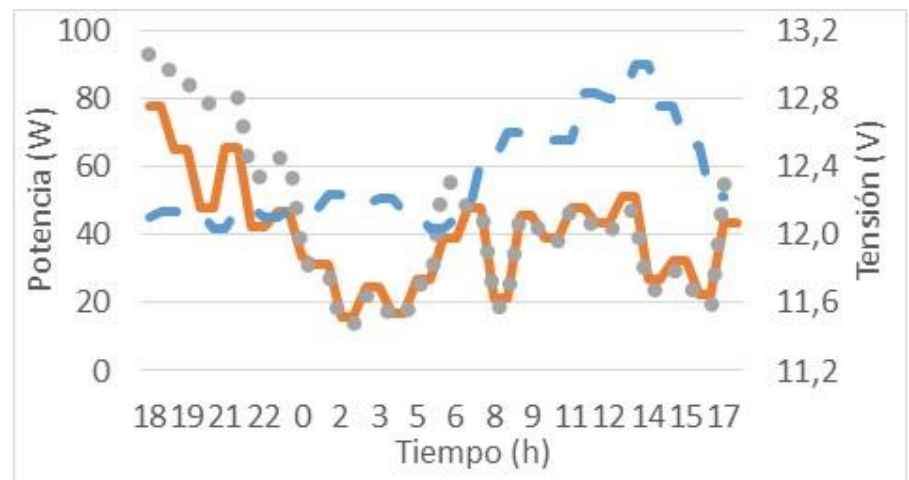

Fig. 10. Potencia demandada estimada (línea punteada) y medida (línea continua), y tensión en el barraje de 12 V (línea discontinua) - Prueba 1. Fuente: autores.

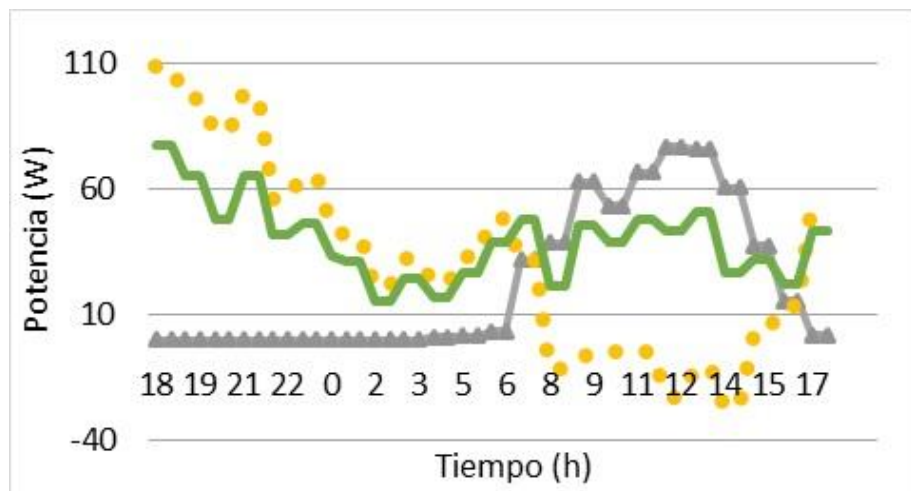

Fig. 11. Potencia desarrollada por dispositivo - Prueba 1. Línea con marcadores (potencia salida SEPIC), línea punteada (potencia batería FV), línea continua (potencia demandada medida). Fuente: autores.

Prueba 2. En esta prueba se empleó una curva de irradiancia preestablecida. Los resultados de esta prueba se observan en la Fig. 12 y Fig. 13. En la Fig. 12 se observa la tensión en el barraje de $24 \mathrm{~V}$ (línea con rombos), la tensión en el barraje de $12 \mathrm{~V}$ (línea con triángulos), y la demanda estimada (línea punteada) y la demanda medida (línea continua).

En la Fig. 13, la línea punteada es la potencia de salida del SEPIC, la línea interrumpida es la potencia en la batería $\mathrm{FV}$, la línea con marcadores es la potencia de salida del Boost del VE y la línea continua es la potencia demandada medida.

Esta prueba empezó las 00:00 h, por lo que inicialmente la demanda fue suplida por las baterías FV. A las 06:00 h la tensión en el barraje de $12 \mathrm{~V}$ cae, pero el nivel de irradiancia es suficiente para asegurar la continuidad del sistema. A las
14:00 h VFv disminuye, por lo que el SGE evalúa la disponibilidad del VE para operar como fuente y permite la conexión de la batería del VE al barraje de $24 \mathrm{~V}$, lo que hace que la batería FV no aporte energía.

Cuando la batería del VE se emplea como alimentación, solo se suple la demanda de las dos cargas de alta prioridad, es decir, el circuito especial y la nevera. El sistema puede volver a su condición de operación normal cuando la energía debida a la irradiancia recargue la batería FV.

Prueba 3. En este caso se empleó una curva de irradiancia con una condición menos crítica que en los casos anteriores. La Fig. 14 presenta el comportamiento del nivel de tensión de $12 \mathrm{~V}$ (línea interrumpida) debido a la inyección de potencia realizada por el sistema FV. La potencia estimada corresponde a la línea 
punteada, mientras que la demanda medida es la línea continua. Para este caso no fue necesario que el sistema de gestión realizara un deslastre de cargas a lo largo del día.

Los cambios después de las 17:00 h se deben a la demanda variable, debido a que el VE se encuentra en estado de recarga. La Fig. 15 presenta la potencia de los dispositivos propios del emulador. La línea punteada es la potencia de salida del SEPIC, la línea continua es la potencia en la batería FV, la línea interrumpida es la potencia demandada medida.

Este caso es un ejemplo de buena operación del sistema, ya que durante todo el día se puede hacer uso de los diferentes circuitos eléctricos, se lleva a cabo la recarga de las baterías mediante el recurso solar, y se cuenta con el VE como carga.

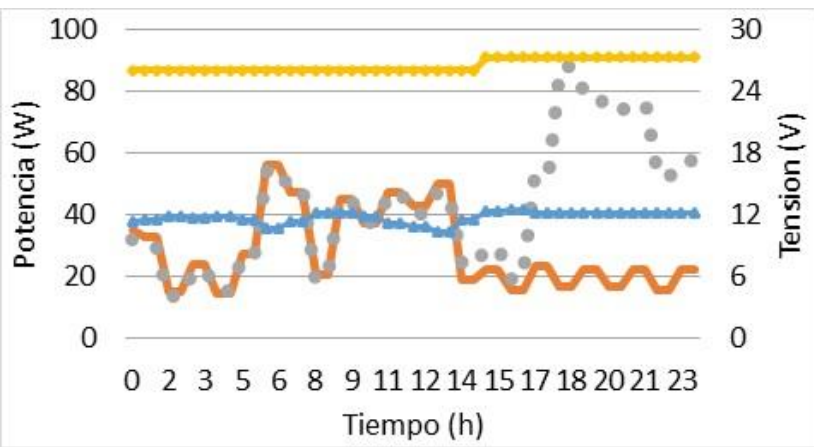

Fig. 12. Potencia demandada estimada (línea punteada) y medida (línea continua), y tensión en los barrajes de $12 \mathrm{~V}$ (línea con triángulos) y $24 \mathrm{~V}$ (línea con rombos) - Prueba 2. Fuente: autores.

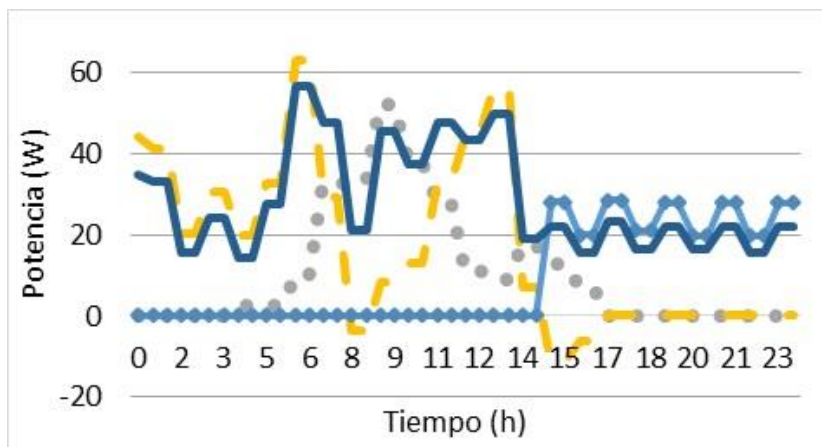

Fig. 13. Potencia desarrollada por dispositivo - Prueba 2. Línea punteada (potencia salida SEPIC), línea interrumpida (potencia batería FV), línea con marcadores (potencia salida VE), línea continua (potencia demandada medida). Fuente: autores.

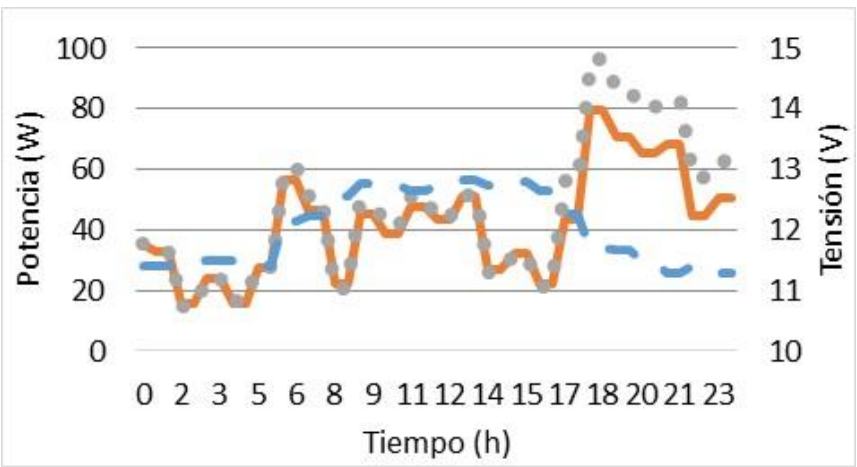

Fig. 14. Potencia demandada estimada (línea punteada) y medida (línea continua), y tensión en el barraje de 12 V (línea discontinua) - Prueba 3. Fuente: autores. 


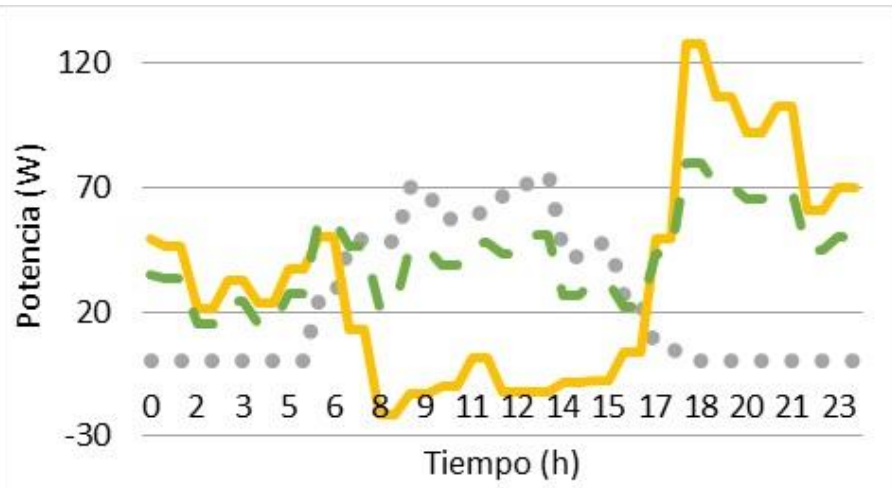

Fig. 15. Potencia desarrollada por dispositivo - Prueba 3. Línea punteada (potencia salida SEPIC), línea continua (potencia batería FV), línea interrumpida (potencia demandada medida). Fuente: autores.

\subsection{Discusión y análisis}

En la literatura es posible encontrar diferentes artículos enfocados a emuladores de micro-redes en diferentes contextos. En [24] se presenta una adecuada revisión y clasificación de los diferentes emuladores de micro-redes eléctricas, según los autores es posible clasificar un emulador de micro-red en uno de los cuatro niveles propuestos, el primer nivel dado a aquellos sistemas que permiten una emulación global; el segundo nivel en donde se contempla una emulación particular para la agregación de cargas, sistemas de almacenamiento y generación; un tercer nivel, en el que se contempla la emulación del recurso; y un cuarto nivel, enfocado a la emulación específica de los componentes, siendo en este nivel en el que se encuentra el emulador que se aborda en el presente artículo. El emulador propuesto emula el recurso fotovoltaico, la tecnología V2G, así como su interacción con la carga y el sistema de almacenamiento. También presenta desde el punto de vista del hardware los diferentes equipos convertidores (Boost, SEPIC e Inversor) con la posibilidad de ser reprogramados para cambiar sus controladores y sus puntos de operación dentro de ciertos rangos permitidos.

Teniendo en cuenta lo anterior, es posible comparar el emulador propuesto con emuladores pertenecientes al mismo nivel. En [25] se propone la construcción de tres emuladores a escala (100 W), cada un conformado por un panel fotovoltaico y una batería como sistema de almacenamiento, la idea del artículo es poder desarrollar diferentes algoritmos de gestión e interacción entre los emuladores. La comunicación entre ellos se lleva a cabo a través de TCP/IP. En [26] se presenta un emulador de una micro-red aislada basada en tres sistemas de generación (geotermia, biogas y fotovoltaico) y su sistema de almacenamiento, el artículo se orienta a dos premisas: la primera al control primario de los equipos (control Droop); y la segunda a la gestión, buscando ante todo igualar la generación con el consumo de la carga y conservar el SOC de la batería.

Aunque los artículos anteriormente presentados realizan grandes aportes desde el punto de vista del desarrollo de los emuladores de las micro-redes, el presente artículo presenta la gran ventaja de incorporar la tecnología V2G y hacer un proceso de emulación en todo el contexto de operación de dicha tecnología (generadorcarga), lo que permite explorar nuevos algoritmos de gestión como el propuesto. Adicionalmente, y como se mencionó en párrafos anteriores, el haber desarrollado los diferentes equipos convertidores, permite ajustarlos de manera individual y proponer nuevos esquemas de control de forma particular y de gestión en todo el 
contexto de la micro-red, la cual tiene un enfoque claramente domiciliario.

Por otra parte, las pruebas realizadas muestran que la respuesta del sistema depende directamente del SOC de las baterías y de la hora en la que comienzan las pruebas. Esto se observó cuando se emuló una irradiancia desfavorable y aun así el sistema solo presentó una interrupción, esto debido a que la batería empleada se encontraba plenamente cargada.

El emulador desarrollado reproduce el comportamiento real de una micro-red residencial. En este sentido los hábitos de consumo del usuario se afectan cuando no se cuenta con un sistema de gestión que lleve a cabo un deslastre de carga y habilite fuentes de respaldo para el suministro de energía.

Adicionalmente, es importante adelantar cambios en los hábitos de consumo para suplir la demanda de cargas que tienen un impacto alto en la curva de demanda, en los periodos donde hay un buen recurso solar, dejando energía disponible para alimentar otras cargas en las horas pico.

\section{CONCLUSIONES}

El emulador de micro-red aislada desarrollado permite realizar estudios acerca del comportamiento e impacto en este tipo de sistemas al conectar baterías FV y EV de diferentes capacidades. Además, con este prototipo es posible evaluar el impacto en la recarga de la batería de un VE y su funcionalidad como tecnología V2G.

A partir de la información acerca de los hábitos de consumo de los usuarios, el SGE atenúa el impacto en el confort del usuario ante un racionamiento de energía.

El consumo del VE está ligado al SOC de la batería, por lo tanto, el impacto dentro del sistema del VE conectado como carga es variable, y debido a esto el deslastre de carga no siempre se realiza en los mismos lapsos de tiempo.

Los tiempos de operación del emulador de la micro-red residencial dependen de las corrientes de las baterías del sistema FV y del VE, por lo tanto, la autonomía y capacidad de almacenamiento están ligadas al tipo de batería seleccionada.

Se desarrolló una herramienta que permite realizar diferentes estudios en micro-redes aisladas, lo que facilita la validación de hipótesis y modelos teóricos.

El emulador propuesto presenta la gran ventaja de incorporar la tecnología V2G y hacer un proceso de emulación en todo el contexto de operación de dicha tecnología, lo que permite incorporar nuevos algoritmos de gestión energética.

\section{AGRADECIMIENTOS}

Esta investigación es financiada por el Centro de Investigaciones y Desarrollo Científico de la Universidad Distrital Francisco José de Caldas - CIDC (Contrato: 2-7-563-2017).

\section{REFERENCIAS}

[1] A. Aggarwal, A. Singhal, and S. J. Darak, "Clean and Green India: Is Solar Energy the Answer?," IEEE Potentials, vol. 37, no. 1, pp. 40-46, Jan. 2018.

[2] L. Meng, E. R. Sanseverino, A. Luna, T. Dragicevic, J. C. Vásquez, and J. M. Guerrero, "Microgrid supervisory controllers and energy management systems: A literature review," Renew. Sustain. Energy Rev., vol. 60, pp. 1263-1273, Jul. 2016.

[3] Xiang Eric Yu, Yanbo Xue, S. Sirouspour, and A. Emadi, "Microgrid and transportation electrification: A review," in 2012 IEEE Transportation Electrification Conference and Expo (ITEC), 2012, pp. 1-6.

[4] C. Shum et al., "The development of a smart grid co-simulation platform and case study on Vehicle-to-Grid voltage support application," in 2013 IEEE International Conference on Smart Grid Communications (SmartGridComm), 2013, pp. 594-599. 
[5] C. C. Mendoza, A. M. Quintero, F. Santamaría, and A. Alarcón, "Coordinated recharge of electric vehicles in real time," DYNA, vol. 83, no. 197, p. 222, Jun. 2016.

[6] C. Mendoza, A. Quintero, F. Santamaría, and A. Alarcon, "Estimation of electric energy required by electric vehicles based on travelled distances in a residential zone," TECCIENCIA, vol. 11, no. 21, pp. 17-24, Sep. 2016.

[7] A. Anvari-Moghaddam, J. M. Guerrero, J. C. Vásquez, H. Monsef, and A. Rahimi-Kian, "Efficient energy management for a grid-tied residential microgrid," IET Gener. Transm. Distrib., vol. 11, no. 11, pp. 2752-2761, Aug. 2017.

[8] T. Sattarpour, D. Nazarpour, and S. Golshannavaz, "A multi-objective HEM strategy for smart home energy scheduling: A collaborative approach to support microgrid operation," Sustain. Cities Soc., vol. 37, pp. 26-33, Feb. 2018.

[9] A. M. Vega, F. Santamaria, and E. Rivas, "Modeling for home electric energy management: A review," Renew. Sustain. Energy Rev., vol. 52, pp. 948-959, Dec. 2015.

[10] I. R. Pinzón Vela, "Estimación de funciones de consumo de energia eléctrica para clientes residenciales en Bogotá ," Pontificia Universidad Javeriana, 2010.

[11] J. P. Lozano Celis and W. C. Guzmán Espitia, "Evaluación de demanda de energía eléctrica según hábitos de consumo actuales en la ciudad de Bogotá," Universidad Distrital Francisco José de Caldas, 2016.

[12] K. A. Hernández Hernández and J. S. Carrillo Cruz, "Análisis de la curva de demanda eléctrica para usuarios residenciales estrato 4 en la ciudad de Bogotá ante diferentes escenarios de los hábitos de consumo," Universidad Distrital Francisco José de Caldas, 2017.

[13] Empresas Públicas de Medellín, "Uso inteligente de la energía eléctrica Banco de recomendaciones," EPM, 2012. [Online]. Available:

https://www.epm.com.co/site/Portals/2/docum entos/banco_de_recomendaciones_uso_intelig ente_energia_electricamarzo_27.pdf.

[14] Universidad Nacional de Colombia, "Determinación del consumo final de energía en los sectores residencial urbano y comercial y determinación de consumos para equipos domésticos de energía eléctrica y gas,” Bogotá, 2006.

[15] Corporación para la Energía y el Medio Ambiente. Corpoema, "Caracterización energética del sector residencial urbano y rural en colombia,” Bogotá, vol.1, 2012.

[16] Corporación para la Energía y el Medio Ambiente. Corpoema, "Caracterización energética del sector residencial urbano y rural en colombia," vol.2, Bogotá, 2012.

[17] A. M. Vega Escobar, "Gestión de la energía eléctrica domiciliaria con base en la gestión activa de la demanda," Universidad Distrital Francisco José de Caldas, 2018.

[18] Instituto Colombiano de Normas Técnicas y Certificación, Código eléctrico colombiano NTC 2050, 1st ed. Bogotá: ICONTEC, 2002.

[19] S. J. Chiang, Hsin-Jang Shieh, and MingChieh Chen, "Modeling and Control of PV Charger System With SEPIC Converter," IEEE Trans. Ind. Electron., vol. 56, no. 11, pp. 4344-4353, Nov. 2009.

[20] H. R. Galiveeti, A. K. Goswami, and N. B. Dev Choudhury, "Impact of plug-in electric vehicles and distributed generation on reliability of distribution systems," Eng. Sci. Technol. an Int. J., vol. 21, no. 1, pp. 50-59, Feb. 2018.

[21] G. A. Putrus, P. Suwanapingkarl, D. Johnston, E. C. Bentley, and M. Narayana, "Impact of electric vehicles on power distribution networks," in 2009 IEEE Vehicle Power and Propulsion Conference, 2009, pp. 827-831.

[22] D. Martínez Vicente, "El impacto del vehículo eléctrico en la red de distribución," Universitat Politécnica de Catalunya, 2011.

[23] J. Kalawoun, K. Biletska, F. Suard, and M. Montaru, "From a novel classification of the battery state of charge estimators toward a conception of an ideal one," J. Power Sources, vol. 279, pp. 694-706, Apr. 2015.

[24] E. Prieto-Araujo, P. Olivella-Rosell, M. Cheah-Mañe, R. Villafafila-Robles, and $\mathrm{O}$. Gomis-Bellmunt, "Renewable energy emulation concepts for microgrids," Renew. Sustain. Energy Rev., vol. 50, pp. 325-345, Oct. 2015.

[25] L. K. Gan, B. Riar, J. Lee, and D. Howey, "Low-cost modular PV-battery microgrid emulator for testing of energy management algorithms," in 2017 IEEE Second International Conference on DC Microgrids (ICDCM), 2017, pp. 602-608.

[26] E. Radu, P. Dorin, P. Toma, and L. Eniko, "An islanded renewable energy microgrid emulator for geothermal, biogas, photovoltaic and lead acid battery storage," in 2017 IEEE 26th International Symposium on Industrial Electronics (ISIE), 2017, pp. 2109-2114. 